\title{
Trans-Umbilical Laparo-Endoscopic Single Site Surgery with Hepatic Ductoplasty in Management of Childhood Choledochal Cyst: A Single Surgeon Experience with 46 Cases
}

\author{
Tran Ngoc Son ${ }^{1,2 *}$, Dinh Anh Duc ${ }^{1}$ and Duong Van Mai ${ }^{2}$ \\ ${ }^{1}$ Department of Surgery, National Hospital of Pediatrics, Vietnam \\ ${ }^{2}$ Department of Pediatric Surgery, Saint Paul Hospital, Vietnam
}

*Corresponding author: Tran Ngoc Son, Associate Professor of Surgery, Deputy

Director, Chief of Department of Pediatric Surgery, Saint Paul Hospital, Vietnam.

\section{Abstract}

Aim: To present our techniques and results of trans-umbilical laparo-endoscopic single site surgery (TULESS) with hepatic ductoplasty in the management of childhood choledochal cyst (ChC).

Methods: All ChC cases undergoing TULESS excision of ChC, ductoplasty and hepatico-jejunostomy by the same surgeon from October 2012 to October 2017 were reviewed. For TULESS, 3 trocars were placed at a single umbilical skin incision and conventional instruments were used.

Results: 46 patients (35 girls, 11 boys) with hepatic ductoplasty were identified from total 237 patients with ChC undergoing TULESS by the same surgeon for the review period. The median age was 17 months. Ductoplasty for a small common hepatic duct (less than $5 \mathrm{~mm}$ ) was carried out in 38 patients and for the presence of an aberrant duct in 8 patients. The median operative time was 195 minutes. There was no intraoperative complication, no conversion to open surgery. Additional trocars (conversion to conventional laparoscopic surgery) were required in just the first case of aberrant duct. Postoperative bile leak was noted in one patient $(2.2 \%)$, which was resolved with non-operative treatment. The median postoperative hospital stay was 5 days. At a median follow up of 36 months (range: 3 months to 60 months), one patient needed redo surgery for anastomotic stenosis; all other patients were in good health, with excellent postoperative cosmesis.

Conclusion: TULESS with conventional instruments is feasible and safe for hepatic ductoplasty for childhood ChC with small common hepatic duct or aberrant duct.

Keywords: Chylous ascites; Intra-abdominal pressure; Pregnancy; Vaginal delivery

\section{Introduction}

Choledochal cyst ( $\mathrm{ChC}$ ) is a relatively common disease in children. Surgical repair is the mainstay in the management of this anomaly since total excision of the cyst and hepatico-enterostomy have been accepted as standard procedures. With the development of the minimally invasive surgical techniques, conventional laparoscopic surgery (CLS) with 4 separate trocars has become popular and has replaced the classic open approach in the management of childhood ChC in many centers nowadays [1-4]. For $\mathrm{ChC}$ in general, good hepatico-intestinal anastomosis is mandatory to minimize of postoperative morbidities such as bile leak and/ or anastomotic stenosis and cholangitis. These complications have been reported to be up to $10 \%$ in different series for both open and laparoscopic approaches [1-9]. To avoid aforementioned complications, especially in cases of unfavorable biliary anatomy such as a very small common hepatic duct or presence of an aberrant duct, a hepatic ductoplasty (HDP) may be necessary [6-10]. Hepatic ductoplasty (HDP) is considered to be a technically demanding procedure and should be performed by surgeons with proficiency and experience [6-8]. Transumbilical laparoscopic single site surgery (TULESS) or SILS (single incision laparoscopic surgery) has been recently introduced to the management of childhood $\mathrm{ChC}$ with promising initial results and cosmetic advantages over the CLS [11-13]. However, surgeon's experience with TULESS is limited and the feasibility of TULESS for complex cases ChC requiring HPD 
has been questioned $[14,15]$. Therefore, we conducted this first study to investigate the feasibility and safety of TULESS for HDP in management of childhood ChC.

\section{Materials and Methods}

All ChC cases undergoing ductoplasty by TULESS with conventional instruments at our centers from October 2012 to October 2017 were reviewed. All the operations were performed by the same surgeon with total excision of the cyst and hepaticojejunostomy. The operative techniques of TULESS for ChC has been described in detail in our previous report [13]. Our indications for hepatic ductoplasty were $\mathrm{ChC}$ cases with diameter of the common hepatic duct diameter of less than $5 \mathrm{~mm}$ or the presence of an aberrant duct. Ductoplasty for common hepatic duct smaller than $5 \mathrm{~mm}$ was performed with a $4-5 \mathrm{~mm}$ longitudinal incision by scissors on the anterior duct wall at 12 o'clock to create larger hepatico-jejunal anastomosis (at least $8-10 \mathrm{~mm}$ ). In the case of an aberrant bile duct, a "double barrel" orifice was created by suture with PDS 6.0 to join the aberrant duct to the common hepatic duct for a single hepatico-jejunal anastomosis. All hepatico-jejunal anastomoses were done with PDS 5.0 single layer running suture. Abdominal drain placement was optional. Patients were discharged after resuming full oral feeding and being asymptomatic. All patients were followed up after discharge by a protocol consisting of clinical examination, liver functional tests and an abdominal ultrasound at intervals of 1 month, 3 months, 6 months intervals and then annually. Patient's characteristics, intraoperative findings, operative time and results were analyzed.

\section{Results}

For the study period, from total 237 patients with ChC undergoing TULESS 46 patients with HDP were identified (19.4\%). There were 35 girls (76.1\%) and 11 boys (23.9\%) with a median age of 17 months (range: 1 month to 11 years). The median bodyweight at surgery is $9.7 \mathrm{~kg}$ (range: 3.4 to $32 \mathrm{~kg}$ ). The median size of ChC is $3.8 \mathrm{~cm}$ (range: $1.5 \mathrm{~cm}$ to $11 \mathrm{~cm}$ ). HDP for small common hepatic duct were carried out in 38 patients. The median hepatic duct diameter is $3.4 \mathrm{~mm}$ (range: $2 \mathrm{~mm}$ to $4.5 \mathrm{~mm}$ ). HDP for aberrant duct was performed in 8 patients. All aberrant ducts were on the right side of the common hepatic duct, with diameter of $1.5 \mathrm{~mm}-2.5 \mathrm{~mm}$ and were discovered during transection of the upper end of the $\mathrm{ChC}$ together with the cystic duct. In 5 cases, the aberrant duct made confluence with the cystic duct. In other 3 cases, the aberrant duct confluent with the common hepatic duct was just next the cystic duct orifice. The median operative time is 195 minutes (range: 150-345 minutes). There are no intraoperative complications, no conversion to open surgery. Additional trocars (conversion to CLS) were needed in just the first case with aberrant duct. The rate of anastomotic complications is $4.4 \%$ with postoperative bile leak in one patient (2.2\%) that resolved with nonoperative management. and hepatico-jejunal anastomotic stenosis 2 months after the operation in another patient (2.2\%). The later patient developed jaundice and cholangitis necessitating redo surgery with normalization of liver function tests and abdominal ultrasound.
Both complications occurred in the HDP patients with a small common hepatic duct. There is no complication in the group of patients with HDP with aberrant duct. The remaining patients recovered well with median postoperative hospital stay of 5 days (range 4 -7 days). At a median follow up of 36 months (range: 3 months to 60 months), all the patients were in good health and without complication. The postoperative cosmesis is excellent as all patients were virtually scarless.

\section{Discussion}

One of the key points in the surgical management of $\mathrm{ChC}$ is performing of hepatico-enterortomy since most common complications after surgery for $\mathrm{ChC}$ such as bile leak or anastomotic stenosis (with subsequent cholangistis and biliary stones formation) were related to this anastomosis [1-9]. After open surgery for ChC, the rate of postoperative complications, especially anastomotic stricture requiring redo surgery, may be up to $10 \%$ [9]. The results of CLS for childhood ChC has been reported to be similar or better than open surgery $[3,4]$ with the postoperative complication rate of $4.6 \%$ to $7.7 \%$ [1-8]. For unfavorable biliary anatomy (such as too small common hepatic duct or presence of an aberrant duct), the complication of bile leak and anastomotic stenosis rate might be higher because of difficulties with the hepatico-intestinal anastomosis. HDP techniques with a widened anastomosis and/ or joining the aberrant duct to the common hepatic duct were recommended by Todani et al. [9] [10]. This technique using conventional laparoscopic HDP has been also reported [6-8].

The initial experience with TULESS or SILS by conventional laparoscopic instruments for childhood $\mathrm{ChC}$ has been reported to be with promising results $[11,13]$. Compared to CLS, the difficulties in TULESS are mainly ergonomic with very limited angulation and collision of the instruments $[14,15]$. A study comparing SILS and CLS showed no significant differences in operative time and results between the two approaches with better postoperative cosmesis for SILS [12]. Our study focused on TULESS in cases with HDP and it showed that TULESS was feasible and for HDP in children. In our series of 46 cases, only the first case of aberrant duct (and it was early in our series of TULESS) was converted to CLS. In all the remaining cases ductoplasty was successfully performed by TULESS, including 7 cases of aberrant duct. Our procedure of ductoplasty did not prolong the operative time with similar operative time as previously reported in TULESS cases [13]. Our rate of ductoplasty for ChC was higher than other report of CLS [6-8]. The reason was that we purposely adopted widening the anastomosis when the common hepatic duct less than $5 \mathrm{~mm}$. Our technique of ductoplasty for small common hepatic duct was similar to the technique described by Li L et al [7]. The different point in doing the hepaticojejunal anastomosis in our TULESS series compared to our previous CLS series [1-2] was that we used running suture in all cases while interrupted sutures were used in CLS cases of common hepatic duct less than $10 \mathrm{~mm}$. The results of this study showed that the running suture combined with ductoplasty gave good results. The rate of anastomotic complications was $4.4 \%$ in this series, similar to other 
reports of ductoplasty by CLS [6-8]. In our experience with both CLS and TULESS with conventional straight instruments, the surgeon can apply almost the same surgical tactics in both approaches for ductoplasty. Carefully gentle handling of biliary duct tissue, and proficiency of laparoscopic suturing are essential to get a good outcome.

\section{Conclusion}

Our TULESS technique with conventional instruments is feasible and safe for hepatic ductoplasty for childhood ChC with small common hepatic duct or aberrant duct. In experienced hands, TULESS can be a good option in the minimally invasive management of childhood $\mathrm{ChC}$, including cases with unfavorable biliary anatomy.

\section{Acknowledgement}

We would like to thank Professor Ai Xuan Holterman for her help in editing of this manuscript.

\section{Conflict of Interest}

No conflict of interest.

\section{References}

1. Nguyen TL, Hien PD, Dung LA, Son TN (2010) Laparoscopic repair for choledochal cyst: lessons learned from 190 cases. J Pediatr Surg 45(3): 540-544.

2. Liem NT, Pham HD, Dung le A, Son TN, Vu HM, et al. (2012) Early and intermediate outcomes of laparoscopic surgery for choledochal cyst with 400 patients. J Laparoendosc Adv Surg Tech A 22(6): 599-603.

3. Liem NT, Pham HD, Vu HM (2011) Is the laparoscopic operation as safe as open operation for choledochal cyst in children. J Laparoendosc Adv Surg Tech A 2011; 21(4): 367-370.

4. Diao M, Li L, Cheng (2011) Laparoscopic versus Open Roux-en-Y hepatojejunostomy for children with choledochal cysts: intermediateterm follow-up results. Surg Endosc 25(5): 1567-1573.
5. Qiao G, Li L, Li S, Tang S, Wang B, et al. (2015) Laparoscopic cyst excision and Roux-Y hepaticojejunostomy for children with choledochal cysts in China: a multicenter study. Surg Endosc 29(1): 140-144.

6. Li S, Wang W, Yu Z, Xu W (2014) Laparoscopically assisted extrahepatic bile duct excision with ductoplasty and a widened hepaticojejunostomy for complicated hepatobiliary dilatation. Pediatr Surg Int 30(6): 593598.

7. Li L, Liu SL, Hou WY, Cui L, Liu XL, et al. (2008) Laparoscopic correction of biliary duct stenosis in choledochal cyst. J Pediatr Surg 43(4): 644646.

8. Urushihara N, Fukuzawa H, Fukumoto K, Sugiyama A, Nagae H, et al. (2011) Totally laparoscopic management of choledochal cyst: Rouxen-Y Jejunojejunostomy and wide hepaticojejunostomy with hilar ductoplasty. J Laparoendosc Adv Surg Tech A 21(4): 361-366.

9. Todani T, Watanabe Y, Toki A, Ogura K, Wang ZQ, et al. (1998) Co-existing biliary anomalies and anatomical variants in choledochal cyst. Br J Surg 85(6): 760-763.

10. Todani T, Watanabe Y, Toki A, Sato Y, Ogura K, et al. (1997) Ductoplasty for an aberrant hepatic duct in a choledochal cyst. Pediatr Surg Int 12(8): 618-619.

11. Diao M, Li L, Dong N, Li Q Cheng W, et al. (2012) Single-incision laparoscopic Roux-en-Y hepaticojejunostomy using conventional instruments for children with choledochal cysts. Surg Endosc 26(6): 1784-1790.

12. Diao M, Li L, Li Q, Ye M, Cheng W, et al. (2013) Single-Incision Versus Conventional Laparoscopic Cyst Excision and Roux-Y Hepaticojejunostomy for Children With Choledochal Cysts: a CaseControl Study. World J Surg 37: 1707-1713.

13. Son TN, Liem NT, Hoan VX (2014) Transumbilical laparoendoscopic single-site surgery with conventional instruments for choledochal cyst in children: early results of 86 cases. J Laparoendosc Adv Surg Tech A 24(12): 907-910.

14. Blanco FC, Kane TD (2012) Single-port laparoscopic surgery in children: concept and controversies of the new technique. Minim Invasive Surg 2012: 232347.

15. Saldaña LJ, Targarona EM (2013) Single-Incision Pediatric Endosurgery: A Systematic Review. J Laparoendosc Adv Surg Tech A 23(5): 467-480. 